\title{
A Large-Update Feasible Interior-Point Algorithm for Convex Quadratic Semi-definite Optimization Based on a New Kernel Function
}

\author{
B. Kheirfam • F. Hasani
}

Received: 16 February 2013 / Revised: 3 July 2013 / Accepted: 16 August 2013 /

Published online: 13 September 2013

(C) Operations Research Society of China, Periodicals Agency of Shanghai University, and

Springer-Verlag Berlin Heidelberg 2013

\begin{abstract}
In this paper we present a large-update primal-dual interior-point algorithm for convex quadratic semi-definite optimization problems based on a new parametric kernel function. The goal of this paper is to investigate such a kernel function and show that the algorithm has the best complexity bound. The complexity bound is shown to be $\mathcal{O}\left(\sqrt{n} \log n \log \frac{n}{\epsilon}\right)$.
\end{abstract}

Keywords Kernel function · Interior-point algorithm · Polynomial complexity · Large-update methods

Mathematics Subject Classification (2010) 90C22 - 90C25 · 90C33

\section{Introduction}

In this paper, we consider the standard form of the convex quadratic semi-definite optimization (CQSDO) problem,

$$
\begin{array}{ll}
\min & C \bullet X+\frac{1}{2} X \bullet \mathcal{Q}(X) \\
\text { s.t. } & A_{i} \bullet X=b_{i}, \quad i=1,2, \cdots, m \\
& X \succeq 0,
\end{array}
$$

B. Kheirfam $(\bowtie) \cdot$ F. Hasani

Department of Mathematics, Azarbaijan Shahid Madani University, Tabriz, Iran

e-mail: b.kheirfam@azaruniv.edu 
and its associated dual problem,

$$
\begin{array}{ll}
\max & b^{T} y-\frac{1}{2} X \bullet \mathcal{Q}(X) \\
\text { s.t. } & \sum_{i=1}^{m} y_{i} A_{i}-\mathcal{Q}(X)+S=C \\
& S \succeq 0,
\end{array}
$$

where each $A_{i} \in \mathbf{S}^{n}$ and they are linearly independent, $b \in \mathbb{R}^{m}, C \in \mathbf{S}^{n}$ and $\mathcal{Q}(X)$ : $\mathbf{S}^{n} \rightarrow \mathbf{S}^{n}$ is a given self-adjoint positive semi-definite linear operation on $\mathbf{S}^{n}$, i.e., for any $A, B \in \mathbf{S}^{n}$, then $\mathcal{Q}(A) \bullet B=A \bullet \mathcal{Q}(B)$ and $\mathcal{Q}(A) \bullet A \geqslant 0$. Here, we use $\mathbf{S}^{n}$ to denote the set of all symmetric $n \times n$ matrices. By $G \bullet H$ we denote the trace of $G^{T} H$.

The CQSDO problem was first appeared in the researches of Kojima et al. [11]. This problem is a generalization of the SDO and convex quadratic optimization (CQO) problem. Some efficient interior-point algorithms have been proposed to CQSDO. For example, Nie et al. [14] presented an interior-point algorithm for CQSDO based on potential reduction approach and obtained an iteration bound $\mathcal{O}\left(n \log \frac{n}{\epsilon}\right)$ in the worst case. They [15] also given a predictor-corrector interior-point algorithm for CQSDO using both Dikin-type and Newton centering steps, whose algorithm has an iteration bound $\mathcal{O}\left(\sqrt{n} \log \frac{n}{\epsilon}\right)$. Toh proposed an inexact primal-dual path-following algorithm for CQSDO in [19]. Bai et al. [8] proposed a different technique for finding a class of search directions. Based on this technique, the authors designed a new primal-dual interior-point algorithm for CQSDO with small-update method, and obtained complexity bound.

Among the variants of interior-point methods (IPMs), the so-called primal-dual path-following methods are most efficient from the computational point of view. There are two types of path-following IPMs. The so-called large-update methods have superior practical performance but relatively weak theoretical results. While the so-called small-update methods enjoy the best known iteration bound but are inefficient at practice. Recently, Peng et al. $[16,17]$ replaced the primal-dual logarithmic barrier by a so-called self-regular barrier function, which is determined by a simple univariate self-regular function, called its kernel function. The search direction was modified accordingly, and a large-update method was obtained for which the theoretical iteration bound is $\mathcal{O}\left(\sqrt{n} \log n \log \frac{n}{\epsilon}\right)$. Bai et al. [2-6, 20] proposed new primal-dual IPMs based on eligible kernel functions. They also proposed a scheme for analyzing the algorithms based on four conditions on the kernel function [2] and obtained an $\mathcal{O}\left(\sqrt{n} \log \frac{n}{\epsilon}\right)$ iteration bound for small-update IPMs in all cases and an $\mathcal{O}\left(\sqrt{n} \log n \log \frac{n}{\epsilon}\right)$ iteration bound for large-update methods with a specific kernel function [6]. Some new analytic tools were developed for the analysis of IPMs based on such kernel functions. As a result the analysis is much simpler than that in $[16,17]$, whereas the iteration bounds are at least as good. Recently, Kheirfam in [10] introduced a trigonometric kernel function and analyzed the feasible primal-dual IPMs for SDO based on this kernel function. He showed that the primal-dual IPMs for solving SDO enjoys $\mathcal{O}\left(n^{\frac{3}{4}} \log \frac{n}{\epsilon}\right)$ as the worst case iteration complexity. Amini and 
Haseli [1] presented an interior-point algorithm for LO based on a new kernel function and obtained the best known iteration bound for large-update methods. Recently, Wang and Zhu [21] proposed a unified kernel function approach to primal-dual IPMs for CQSDO based on kernel functions which were previously introduced for LO in [2]. Later on, Zhang [23] presented a large-update interior-point algorithm for CQSDO based on a new kernel function and obtained its iteration complexity as $\mathcal{O}\left(\sqrt{n}(\log n)^{2} \log \frac{n}{\epsilon}\right)$ for the CQSDO problems.

Motivated by the complexity results based on kernel functions, we present a largeupdate primal-dual interior-point algorithm for CQSDO based on a new parametric kernel function

$$
\psi(t)=\frac{t^{2}-1}{2}+\frac{t}{q}\left(e^{q\left(\frac{1}{t}-1\right)}-1\right), \quad q \geqslant 2, t>0,
$$

where $q$ is a parameter. Our goal is to deal with the primal-dual interior-point algorithm for CQSDO based on the kernel function $\psi(t)$ as defined by (1.1). Finally, we derive the currently best known iteration bound for the algorithm with large-update method, namely, $\mathcal{O}\left(\sqrt{n} \log n \log \frac{n}{\epsilon}\right)$.

The paper is organized as follows: In Sect. 2 we recall some well-known results on matrices and matrix functions. In Sect. 3 we study the central path and the new search directions for CQSDO. The generic primal-dual interior-point algorithm for CQSDO is also presented. In Sect. 4 we develop some properties of the kernel function $\psi(t)$ and the corresponding barrier function $\Psi(V)$. In Sect. 5 we analyze the algorithm and derive the currently best known iteration bound with large-update method. We present a numerical example in Sect. 6. Finally, some conclusions and remarks follow in Sect. 7.

\section{Preliminaries}

Here, we outline some needed main results on matrices and matrix functions. For a comprehensive study, the reader is referred to [9].

Theorem 2.1 (Spectral Theorem for Symmetric Matrices) The real $n \times n$ matrix $A$ is symmetric if and only if there exists a matrix $Q \in \mathbb{R}^{n \times n}$ such that $Q^{T} Q=I$ and $Q^{T} A Q=\Lambda$ where $\Lambda$ is a diagonal matrix.

Due to Theorem 2.1, let $Q_{V} \in \mathbb{R}^{n \times n}$ be an orthogonal matrix that diagonalizes $V \in$ $\mathbf{S}_{++}^{n}$, i.e., $V=Q_{V}^{T} \operatorname{diag}\left(\lambda_{1}(V), \cdots, \lambda_{n}(V)\right) Q_{V}$ and $Q_{V}^{T} Q_{V}=I$, where $I$ denotes the identity matrix. Then, for the real function $\psi(t), t \in \mathbb{R}_{+}$, the matrix function $\psi: \mathbf{S}_{++}^{n} \rightarrow \mathbf{S}_{++}^{n}$ and the real valued matrix function $\Psi: \mathbf{S}_{++}^{n} \rightarrow \mathbb{R}_{+}$are defined by

$$
\begin{aligned}
& \psi(V)=Q_{V}^{T} \operatorname{diag}\left(\psi\left(\lambda_{1}(V)\right), \cdots, \psi\left(\lambda_{n}(V)\right)\right) Q_{V}, \\
& \Psi(V):=\operatorname{Tr}(\psi(V))=\sum_{i=1}^{n} \psi\left(\lambda_{i}(V)\right)
\end{aligned}
$$


where $\mathbf{S}_{++}^{n}$ denotes the set of all symmetric positive definite $n \times n$ matrices and $\mathrm{Tr}$ stands for the trace operator.

Since the derivatives $\psi^{\prime}(t)$ and $\psi^{\prime \prime}(t)$ are well defined, we can obtain the matrix functions $\psi^{\prime}(V)$ and $\psi^{\prime \prime}(V)$ if $\psi\left(\lambda_{i}(V)\right)$ in (2.2) is replaced by $\psi^{\prime}\left(\lambda_{i}(V)\right)$ and $\psi^{\prime \prime}\left(\lambda_{i}(V)\right)$ for each $i$, respectively.

Definition 2.1 A matrix $M(t)$ is said to be a matrix of function (or a matrix-valued function) if each entry of $M(t)$ is a function of $t$, i.e., $M(t)=\left[M_{i j}(t)\right]$.

The usual concepts of continuity, differentiability, and integrability can be naturally extended to matrix-valued functions, by interpreting them component-wise. Let $M(t)$ and $N(t)$ be two matrices of functions. Then, we have

$$
\begin{aligned}
\frac{d}{d t} M(t) & =M^{\prime}(t), \\
\frac{d}{d t} \operatorname{Tr}(M(t)) & =\operatorname{Tr}\left(M^{\prime}(t)\right), \\
\frac{d}{d t} \operatorname{Tr}(\psi(M(t))) & =\operatorname{Tr}\left(\psi\left(M^{\prime}(t)\right) M^{\prime}(t)\right), \\
\frac{d}{d t}(M(t) N(t)) & =M^{\prime}(t) N(t)+M(t) N^{\prime}(t) .
\end{aligned}
$$

\section{IPMs for CQSDO}

We assume that both (P) and (D) satisfy the interior-point condition (IPC), i.e., there exist $X^{0}, S^{0} \succ 0$ such that

$$
A_{i} \bullet X^{0}=b_{i}, \quad i=1,2, \cdots, m, \quad \sum_{i=1}^{m} y_{i}^{0} A_{i}-\mathcal{Q}\left(X^{0}\right)+S^{0}=C
$$

If the IPC holds, then the optimality conditions for the problems (P) and (D) are (Theorem 4 in [15]):

$$
\begin{aligned}
A_{i} \bullet X & =b_{i}, \quad i=1,2, \cdots, m, X \succeq 0, \\
\sum_{i=1}^{m} y_{i} A_{i}-\mathcal{Q}(X)+S & =C, \quad S \succeq 0, \\
X S & =0 .
\end{aligned}
$$

The basic idea of primal-dual IPMs is to replace the third equation in (3.1), the socalled complementarity condition for (P) and (D), by the parameterized equation 
$X S=\mu I$, with $\mu>0$. Thus, one may consider

$$
\begin{aligned}
A_{i} \bullet X & =b_{i}, \quad i=1,2, \cdots, m, X \succeq 0, \\
\sum_{i=1}^{m} y_{i} A_{i}-\mathcal{Q}(X)+S & =C, \quad S \succeq 0, \\
X S & =\mu I .
\end{aligned}
$$

Since the matrices $A_{i}$ are linearly independent and the IPC holds, the parameterized system (3.2) has a unique solution for each $\mu>0$, denoted by $(X(\mu), y(\mu), S(\mu))$. We call $X(\mu)$ the $\mu$-center of (P) and $(y(\mu), S(\mu))$ the $\mu$-center of (D). The set of $\mu$-centers (with $\mu$-running through positive real numbers) gives a homotopy path which is called the central path of (P) and (D). If $\mu \rightarrow 0$, then the limit of the central path exists and since the limit point satisfies the complementarity condition $X S=0$, the limit yields an optimal solution for (P) and (D).

At a given feasible iterate $(X, y, S)$ with $X \succ 0$ and $S \succ 0$, the search direction $(\Delta X, \Delta y, \Delta S)$ at the current iteration, to follow the Newton approach and to linearize the third equation in (3.2), is the unique solution of the following system:

$$
\begin{aligned}
A_{i} \bullet \Delta X & =0, \quad i=1,2, \cdots, m, \\
\sum_{i=1}^{m} \Delta y_{i} A_{i}-\mathcal{Q}(\Delta X)+\Delta S & =0, \\
X \Delta S+\Delta X S & =\mu I-X S .
\end{aligned}
$$

The system (3.3) can be rewritten as

$$
\begin{aligned}
A_{i} \bullet \Delta X & =0, \quad i=1,2, \cdots, m, \\
\sum_{i=1}^{m} \Delta y_{i} A_{i}-\mathcal{Q}(\Delta X)+\Delta S & =0, \\
\Delta X+X \Delta S S^{-1} & =\mu S^{-1}-X .
\end{aligned}
$$

It is clear that $\Delta S$ is symmetric due to the second equation in (3.4). However, a crucial observation is that $\Delta X$ is not necessarily symmetric because $X \Delta S S^{-1}$ may be not symmetric. Many researchers have proposed methods for symmetrizing the third equation in the Newton system (3.4) such that the resulting new system has a unique symmetric solution. In this paper, we use the Nesterov-Todd symmetrization scheme $[12,13]$ which leads us to the so-called NT-direction. Let

$$
P:=X^{\frac{1}{2}}\left(X^{\frac{1}{2}} S X^{\frac{1}{2}}\right)^{\frac{-1}{2}} X^{\frac{1}{2}}\left[=S^{\frac{-1}{2}}\left(S^{\frac{1}{2}} X S^{\frac{1}{2}}\right)^{\frac{1}{2}} S^{\frac{-1}{2}}\right]
$$

and $D=P^{\frac{1}{2}}$, where for any symmetric positive definite matrix $G$, the exponent $G^{\frac{1}{2}}$ denotes its symmetric square root. The matrix $D$ can be used to scale $X$ and $S$ to the 
same matrix $V$ as follows:

$$
V:=\frac{1}{\sqrt{\mu}} D^{-1} X D^{-1}\left[=\frac{1}{\sqrt{\mu}} D S D\right] .
$$

It follows that

$$
V^{2}=\frac{1}{\mu} D^{-1} X S D .
$$

Note that the matrices $D$ and $V$ are symmetric and positive definite. In NTsymmetrization scheme, the term $X \Delta S S^{-1}$ in the third equation of (3.4) is replaced by $P \Delta S P^{T}$. Thus the system (3.4) becomes

$$
\begin{aligned}
A_{i} \bullet \Delta X & =0, \quad i=1,2, \cdots, m, \\
\sum_{i=1}^{m} \Delta y_{i} A_{i}-\mathcal{Q}(\Delta X)+\Delta S & =0, \\
\Delta X+P \Delta S P^{T} & =\mu S^{-1}-X .
\end{aligned}
$$

Obviously, now $\Delta X$ is a symmetric matrix and system (3.8) still has a unique solution (see [22]).

Let us further define

$$
D_{X}:=\frac{1}{\sqrt{\mu}} D^{-1} \Delta X D^{-1} ; \quad D_{S}:=\frac{1}{\sqrt{\mu}} D \Delta S D .
$$

Then it follows from (3.8) that the scaled NT-search directions $\left(D_{X}, \Delta y, D_{S}\right)$ are obtained from the system

$$
\begin{aligned}
\bar{A}_{i} \bullet D_{X} & =0, \quad i=1,2, \cdots, m, \\
\sum_{i=1}^{m} \Delta y_{i} \bar{A}_{i}-\overline{\mathcal{Q}}\left(D_{X}\right)+D_{S} & =0, \\
D_{X}+D_{S} & =V^{-1}-V,
\end{aligned}
$$

where

$$
\bar{A}_{i}:=\frac{1}{\sqrt{\mu}} D A_{i} D, \quad \overline{\mathcal{Q}}\left(D_{X}\right):=D \mathcal{Q}\left(D D_{X} D\right) D .
$$

So far, we have described the scheme that defines the classical NT-direction. Note that the right-hand side of the third equation in (3.10) is the negative gradient of the matrix barrier function $\Psi_{c}(V)$ with the classical kernel function $\psi_{c}(t)=\frac{t^{2}-1}{2}-\log (t)$, while $\psi_{c}(t)$ satisfies

$$
\begin{aligned}
\psi_{c}^{\prime}(1) & =\psi_{c}(1)=0, \\
\psi_{c}^{\prime \prime}(t) & >0, \quad t>0, \\
\lim _{t \rightarrow 0^{+}} \psi_{c}(t) & =\lim _{t \rightarrow+\infty} \psi_{c}(t)=+\infty .
\end{aligned}
$$


We replace the right-hand-side of the third equation in (3.10) by $-\nabla \Psi(V)$, where $-\nabla \Psi(V)$ is the negative gradient of the matrix barrier function $\Psi(V)$ with the kernel function (1.1). Thus this system can be rewritten as

$$
\begin{aligned}
\bar{A}_{i} \bullet D_{X} & =0, \quad i=1,2, \cdots, m, \\
\sum_{i=1}^{m} \Delta y_{i} \bar{A}_{i}-\overline{\mathcal{Q}}\left(D_{X}\right)+D_{S} & =0, \\
D_{X}+D_{S} & =-\nabla \Psi(V) .
\end{aligned}
$$

The new search direction $\left(D_{X}, \Delta y, D_{S}\right)$ is obtained by solving (3.12) so that ( $\Delta X, \Delta y, \Delta S)$ is computed via (3.9). By taking a step along the search directions with the step size $\alpha$ defined by some line searches, we can construct a new iterate $\left(X_{+}, y_{+}, S_{+}\right)$according to

$$
X_{+}=X+\alpha \Delta X, \quad y_{+}=y+\alpha \Delta y, \quad S_{+}=S+\alpha \Delta S \text {. }
$$

Since $\mathcal{Q}($.$) is self-adjoint positive semi-definite linear operation, we have$

$$
D_{X} \bullet D_{S}=\left(\frac{1}{\sqrt{\mu}} D^{-1} \Delta X D^{-1}\right) \bullet\left(\frac{1}{\sqrt{\mu}} D \Delta S D\right)=\frac{1}{\mu} \Delta X \bullet \mathcal{Q}(\Delta X) \geqslant 0 \text {. }
$$

Note that it is one of the difference between the CQSDO problem and the SDO problem in the analysis of our algorithm.

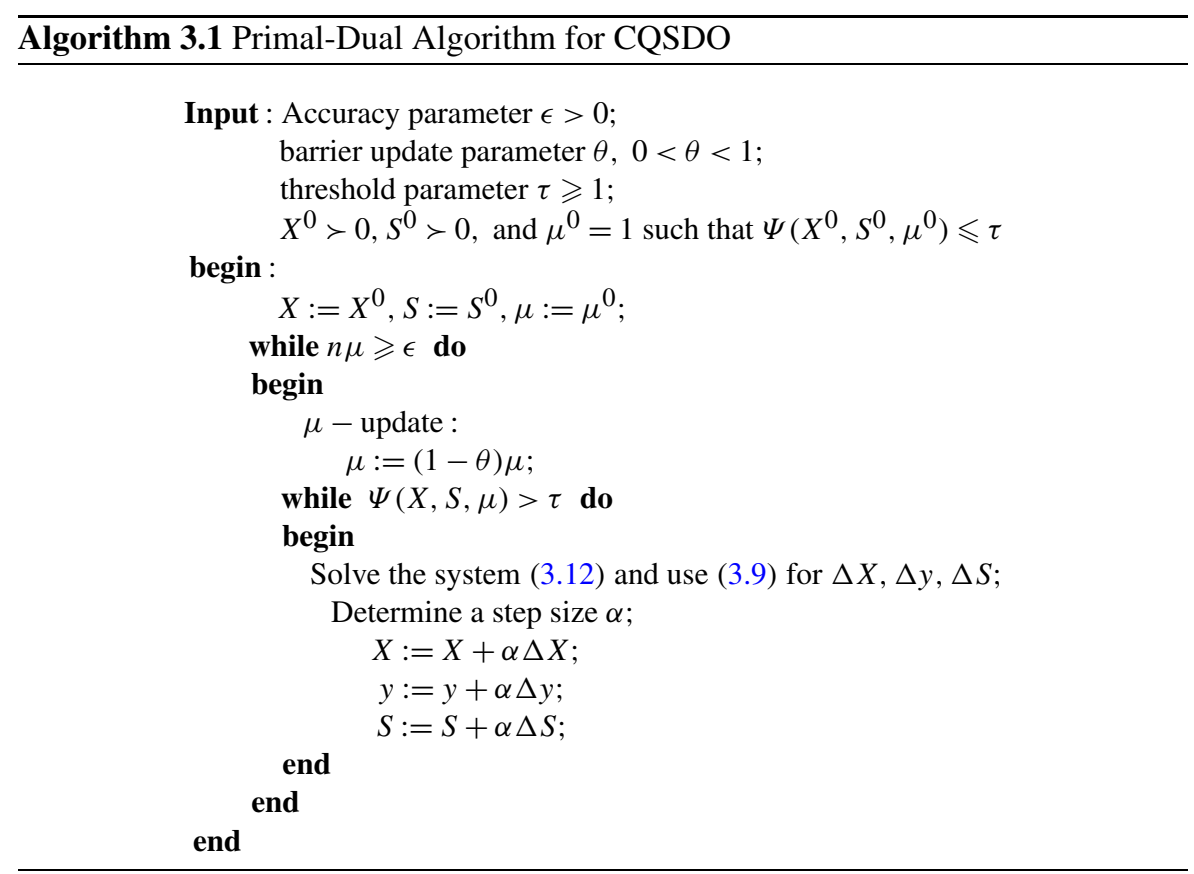


Suppose that the current iterate $(X, y, S)$ is in $\tau$-neighborhood of the corresponding $\mu$-center; i.e., $\Psi(V) \leqslant \tau$. Then, $\mu$ is reduced by the factor $1-\theta$ with $0<\theta<1$, which changes the value of $V$ according to (3.6) and defines a new $\mu$-center $(X(\mu), y(\mu), S(\mu))$. This is the cause that $\Psi(V) \geqslant \tau$. Now we start the inner iteration by calculating new iterate (3.13) where $\Delta X$ and $\Delta S$ are calculated from (3.12) and (3.9) and the step size $\alpha$ is chosen appropriately with the goal of reducing the value of barrier function $\Psi(V)$. If necessary, the procedure is repeated until we find the iterate that again belongs to the $\tau$-neighborhood of the current $\mu$-center, that is, until $\Psi(V) \leqslant \tau$. At this point we start a new outer iteration by reducing the value of $\mu$ again. This process is repeated until $\mu$ is small enough, say until $n \mu \leqslant \epsilon$ for a certain accuracy parameter $\epsilon$, at this stage we have found an $\epsilon$-approximate solution of (P) and (D). The generic IPM outlined above is summarized in Algorithm 3.1.

The parameters $\tau, \theta$ and the step size $\alpha$ play an important role in the algorithm. These parameters should be chosen in such a way that the algorithm is optimized in the sense that the number of iterations required by the algorithm is as small as possible. Usually, if $\theta$ is a constant independent of the dimension of the problem, for instance $\theta=\frac{1}{2}$, then we call the algorithm a large-update method.

\section{Properties of the New Kernel (Barrier) Function}

In this section, we present some basic properties of the kernel function (1.1) and the corresponding barrier function $\Psi(V)$. For $\psi$ we have the first three derivatives as follows:

$$
\begin{aligned}
\psi^{\prime}(t) & =t+\left(\frac{1}{q}-\frac{1}{t}\right) e^{q\left(\frac{1}{t}-1\right)}-\frac{1}{q}, \\
\psi^{\prime \prime}(t) & =1+\frac{q}{t^{3}} e^{q\left(\frac{1}{t}-1\right)}, \\
\psi^{\prime \prime \prime}(t) & =-\frac{q(3 t+q)}{t^{5}} e^{q\left(\frac{1}{t}-1\right)}<0 .
\end{aligned}
$$

The next lemma is essential in the analysis of the algorithm.

Lemma 4.1 For $\psi(t)$ defined in (1.1), we have

$$
\begin{aligned}
\psi^{\prime \prime}(t) & >1, \\
t \psi^{\prime \prime}(t)+\psi^{\prime}(t) & >0, \\
t \psi^{\prime \prime}(t)-\psi^{\prime}(t) & >0, \\
\psi^{\prime \prime \prime}(t) & <0 .
\end{aligned}
$$

Proof Clearly, the relation (4.2) follows (4.4). For the proof of (4.5), consider two cases:

Case 1. Assume that $0<t<1$. 
By using (4.1), (4.2), and $e^{z} \geqslant 1+z, \forall z$, we have

$$
\begin{aligned}
t \psi^{\prime \prime}(t)+\psi^{\prime}(t) & =2 t+\left(\frac{1}{q}-\frac{1}{t}+\frac{q}{t^{2}}\right) e^{q\left(\frac{1}{t}-1\right)}-\frac{1}{q} \\
& \geqslant 2 t+\left(\frac{1}{q}-\frac{1}{t}+\frac{q}{t^{2}}\right)\left(1+q\left(\frac{1}{t}-1\right)\right)-\frac{1}{q} \\
& =2 t+\frac{q}{t}-1+\frac{q^{2}}{t^{2}}\left(\frac{1}{t}-1\right)>2 t>0 .
\end{aligned}
$$

Case 2. Assume that $t \geqslant 1$.

Then $\psi^{\prime}(1)=0$ and using (4.4), we see that $\psi^{\prime}(t)$ is strictly increasing. Hence

$$
t \psi^{\prime \prime}(t)+\psi^{\prime}(t)>t+\psi^{\prime}(t)>t+\psi^{\prime}(1)=t>0 .
$$

The two cases together prove (4.5).

To prove (4.6), by using (4.1) and (4.2) we have

$$
t \psi^{\prime \prime}(t)-\psi^{\prime}(t)=\left(\frac{q}{t^{2}}-\frac{1}{q}+\frac{1}{t}\right) e^{q\left(\frac{1}{t}-1\right)}+\frac{1}{q} .
$$

Define

$$
f(t)=\left(\frac{q}{t^{2}}-\frac{1}{q}+\frac{1}{t}\right) e^{q\left(\frac{1}{t}-1\right)}+\frac{1}{q} .
$$

We have

$$
f^{\prime}(t)=-\left(\frac{3 q t+t^{2}+q^{2}}{t^{4}}\right) e^{q\left(\frac{1}{t}-1\right)}<0 .
$$

This implies that $f(t)$ is strictly decreasing and hence $f(t)>\lim _{t \rightarrow \infty} f(t)=\frac{1}{q}(1-$ $\left.e^{-q}\right)>0$. This completes the proof.

It follows that $\psi(1)=\psi^{\prime}(1)=0$. It is also quite straightforward that

$$
\lim _{t \rightarrow 0^{+}} \psi(t)=\lim _{t \rightarrow+\infty} \psi(t)=+\infty
$$

Due to conditions $\psi^{\prime}(1)=\psi(1)=0$, one can describe the function $\psi(t)$ by its second derivative according to

$$
\psi(t)=\int_{1}^{t} \int_{1}^{\xi} \psi^{\prime \prime}(\zeta) d \zeta d \xi
$$

In the analysis of the algorithm, we also use the norm-based proximity measure defined by

$$
\delta:=\delta(V)=\frac{1}{2}\|\nabla \Psi(V)\|=\frac{1}{2} \sqrt{\sum_{i=1}^{n} \psi^{\prime}\left(\lambda_{i}(V)\right)^{2}} .
$$


The next lemma is very useful in the analysis of interior-point algorithms based on the kernel functions (see for example $[2,16]$ ).

Lemma 4.2 (Lemma 2.1.2 in [17]) Let $\psi(t)$ be a twice differentiable function for $t>0$. Then the following three properties are equivalent:

(i) $\psi\left(\sqrt{t_{1} t_{2}}\right) \leqslant \frac{1}{2}\left(\psi\left(t_{1}\right)+\psi\left(t_{2}\right)\right)$ for $t_{1}, t_{2}>0$.

(ii) $\psi^{\prime}(t)+t \psi^{\prime \prime}(t) \geqslant 0, t>0$.

(iii) $\psi\left(e^{\xi}\right)$ is convex.

Following [17], the property described in Lemma 4.2 is called exponential convexity, or shortly $e$-convexity. Therefore, Lemma 4.2 and (4.5) show that our new kernel function (1.1) is $e$-convex for $t>0$.

Lemma 4.3 (Lemma 9 in [10]) Let the kernel function $\psi(t)$ be defined as in (1.1). Then, we have

$$
\frac{1}{2}(t-1)^{2} \leqslant \psi(t) \leqslant \frac{1}{2} \psi^{\prime}(t)^{2}
$$

Lemma 4.4 Let $\varrho:[0, \infty) \rightarrow[1, \infty)$ be the inverse function of $\psi(t)$ for $t \geqslant 1$. Then

$$
\sqrt{1+2 s} \leqslant \varrho(s) \leqslant 1+\sqrt{2 s} .
$$

Proof The inverse function of $\psi(t)$ for $t \geqslant 1$ is obtained by solving $t$ from

$$
\psi(t)=\frac{t^{2}-1}{2}+\frac{t}{q}\left(e^{q\left(\frac{1}{t}-1\right)}-1\right)=s, \quad t \geqslant 1 .
$$

For $t \geqslant 1, e^{q\left(\frac{1}{t}-1\right)} \leqslant 1$. One has $\frac{t}{q}\left(e^{q\left(\frac{1}{t}-1\right)}-1\right) \leqslant 0$. Therefore,

$$
\frac{t^{2}-1}{2} \geqslant s
$$

this implies that $t=\varrho(s) \geqslant \sqrt{1+2 s}$. This proves the first inequality. For the proof of second inequality, by Lemma 4.3 , we have

$$
s=\psi(t) \geqslant \frac{1}{2}(t-1)^{2},
$$

whence

$$
t=\varrho(s) \leqslant 1+\sqrt{2 s} .
$$

This completes the proof.

The next result, establishes a lower bound of $\delta(V)$ in terms of $\Psi(V)$. 
Lemma 4.5 Let $V \in \mathbf{S}_{++}^{n}$. Then

$$
\delta(V) \geqslant \sqrt{\frac{\Psi(V)}{2}} .
$$

Proof Using (4.9), the second inequality of Lemma 4.3 and (2.2), we have

$$
\begin{aligned}
\delta(V) & =\frac{1}{2} \sqrt{\sum_{i=1}^{n} \psi^{\prime}\left(\lambda_{i}(V)\right)^{2}} \geqslant \frac{1}{2} \sqrt{\sum_{i=1}^{n} 2 \psi\left(\lambda_{i}(V)\right)}=\frac{1}{\sqrt{2}} \sqrt{\sum_{i=1}^{n} \psi\left(\lambda_{i}(V)\right)} \\
& =\sqrt{\frac{\Psi(V)}{2}}
\end{aligned}
$$

which is the desired inequality.

Corollary 4.1 If $\Psi(V) \geqslant 1$, then $\delta(V) \geqslant \frac{1}{\sqrt{2}}$.

At the start of each outer iteration, just before the update of $\mu$ with the factor $1-\theta$, we have $\Psi(V) \leqslant \tau$. Due to the update of $\mu$ the matrix $V$, defined by (3.6), is divided by the factor $\sqrt{1-\theta}$, with $0<\theta<1$, which leads to an increasing in the value of $\Psi(V)$. Then, during the inner iterations, $\Psi(V)$ decreases until it passes the threshold $\tau$ again. Hence, during the course of the algorithm the largest values of $\Psi(V)$ occur just after the updates of $\mu$. In the rest this section, we derive an estimate for the effect of a $\mu$-update on the value of $\Psi(V)$. We start with an important theorem. This is due to the fact that $\psi(t)$ satisfies (4.6) and (4.7).

Theorem 4.1 (Theorem 3.1 in [7]) Let $\varrho$ be the inverse function of $\psi(t)$ for $t \geqslant 1$. Then for any positive definite matrix $V$, and any $\beta \geqslant 1$,

$$
\Psi(\beta V) \leqslant n \psi\left(\beta \varrho\left(\frac{\Psi(V)}{n}\right)\right) .
$$

An immediate consequence of Theorem 4.1 for the real valued matrix function $\Psi(V)$ is given by the following lemma.

Corollary 4.2 Let $0 \leqslant \theta<1$ and $V_{+}=\frac{V}{\sqrt{1-\theta}}$. If $\Psi(V) \leqslant \tau$, then

$$
\Psi\left(V_{+}\right) \leqslant \frac{n \theta+2 \tau+\sqrt{8 n \tau}}{2(1-\theta)} .
$$

Proof Since $\frac{1}{\sqrt{1-\theta}} \geqslant 1$ and $\varrho\left(\frac{\Psi(V)}{n}\right) \geqslant 1$, we have $\frac{1}{\sqrt{1-\theta}} \varrho\left(\frac{\Psi(V)}{n}\right) \geqslant 1$. Using Theorem 4.1 with $\beta=\frac{1}{\sqrt{1-\theta}}$ and the function $\varrho$ is monotonically increasing since $\psi(t)$ for $t \geqslant 1$ is monotonically increasing because of its definition, we have

$$
\Psi\left(V_{+}\right) \leqslant n \psi\left(\frac{1}{\sqrt{1-\theta}} \varrho\left(\frac{\Psi(V)}{n}\right)\right) \leqslant n \psi\left(\frac{\varrho\left(\frac{\tau}{n}\right)}{\sqrt{1-\theta}}\right) \leqslant n \psi\left(\frac{1+\sqrt{\frac{2 \tau}{n}}}{\sqrt{1-\theta}}\right) .
$$


According to the proof of Lemma 4.4, it is clear that $\psi(t) \leqslant \frac{t^{2}-1}{2}$ for $t \geqslant 1$. Therefore, we get

$$
\Psi\left(V_{+}\right) \leqslant \frac{n}{2}\left(\left(\frac{1+\sqrt{\frac{2 \tau}{n}}}{\sqrt{1-\theta}}\right)^{2}-1\right)=\frac{n \theta+2 \tau+\sqrt{8 n \tau}}{2(1-\theta)}
$$

\section{Analysis of the Algorithm}

In this section, we determine a default step size and obtain an upper bound to the decrease of the barrier function $\Psi(V)$ during an inner iteration.

\subsection{Decrease the Value of $\psi(V)$ and Choose a Default Step Size $\alpha$}

In each iteration the search directions $\Delta X, \Delta y$ and $\Delta S$ are obtained by solving the system (3.12) and via (3.9). After a step with size $\alpha$ and due to (3.9), the new iterate is obtained by

$$
X_{+}=X+\alpha \Delta X=X+\alpha \sqrt{\mu} D D_{X} D=\sqrt{\mu} D\left(V+\alpha D_{X}\right) D,
$$

and

$$
S_{+}=S+\alpha \Delta S=S+\alpha \sqrt{\mu} D^{-1} D_{S} D^{-1}=\sqrt{\mu} D^{-1}\left(V+\alpha D_{S}\right) D^{-1} .
$$

According to (3.6), we have

$$
V_{+}=\frac{1}{\sqrt{\mu}}\left[D^{-1} X_{+} S_{+} D\right]^{\frac{1}{2}}
$$

Therefore, $V_{+}^{2}$ is similar to the matrix $\frac{1}{\mu} X_{+}^{\frac{1}{2}} S_{+} X_{+}^{\frac{1}{2}}$ and thus to $\left(V+\alpha D_{X}\right)^{\frac{1}{2}}(V+$ $\left.\alpha D_{S}\right)\left(V+\alpha D_{X}\right)^{\frac{1}{2}}$. Consequently, the eigenvalues of the matrix $V_{+}$are the same as those of $\left[\left(V+\alpha D_{X}\right)^{\frac{1}{2}}\left(V+\alpha D_{S}\right)\left(V+\alpha D_{X}\right)^{\frac{1}{2}}\right]^{\frac{1}{2}}$. Since the proximity after one step is defined by $\Psi\left(V_{+}\right)$, it follows from (2.2) that

$$
\Psi\left(V_{+}\right)=\Psi\left(\left[\left(V+\alpha D_{X}\right)^{\frac{1}{2}}\left(V+\alpha D_{S}\right)\left(V+\alpha D_{X}\right)^{\frac{1}{2}}\right]^{\frac{1}{2}}\right) .
$$

Hence, by Lemma 4.2,

$$
\Psi\left(V_{+}\right) \leqslant \frac{1}{2}\left(\Psi\left(V+\alpha D_{X}\right)+\Psi\left(V+\alpha D_{S}\right)\right) .
$$

Let us denote the difference between the proximity before and after one step by a function of the step size, that is,

$$
f(\alpha):=\Psi\left(V_{+}\right)-\Psi(V) .
$$

Then $f(\alpha) \leqslant f_{1}(\alpha)$, where

$$
f_{1}(\alpha):=\frac{1}{2}\left(\Psi\left(V+\alpha D_{X}\right)+\Psi\left(V+\alpha D_{S}\right)\right)-\Psi(V) .
$$


Obviously

$$
f(0)=f_{1}(0)=0 .
$$

By using (2.4)-(2.6), the derivative of $f_{1}(\alpha)$ with respect to $\alpha$ is given by

$$
f_{1}^{\prime}(\alpha)=\frac{1}{2} \operatorname{Tr}\left(\psi^{\prime}\left(V+\alpha D_{X}\right) D_{X}+\psi^{\prime}\left(V+\alpha D_{S}\right) D_{S}\right) .
$$

This, using (4.9) and the third equation of (3.12), gives

$$
f_{1}^{\prime}(0)=\frac{1}{2} \operatorname{Tr}\left(\psi^{\prime}(V)\left(D_{X}+D_{S}\right)\right)=\frac{1}{2} \operatorname{Tr}\left(-\psi^{\prime}(V)^{2}\right)=-2 \delta(V)^{2} .
$$

If we differentiate $f_{1}^{\prime}$ with respect to $\alpha$, then we obtain

$$
f_{1}^{\prime \prime}(\alpha)=\frac{1}{2} \operatorname{Tr}\left(\psi^{\prime \prime}\left(V+\alpha D_{X}\right) D_{X}^{2}+\psi^{\prime \prime}\left(V+\alpha D_{S}\right) D_{S}^{2}\right) .
$$

In what follows, we use the short notation $\delta:=\delta(V)$ and state four important lemmas without proofs. These are due to the fact that $\psi^{\prime \prime}(t)$ is monotonically decreasing.

Lemma 5.1 (Lemma 4.2 in [20]) One has

$$
f_{1}^{\prime \prime}(\alpha) \leqslant 2 \delta^{2} \psi^{\prime \prime}\left(\lambda_{\min }(V)-2 \alpha \delta\right) .
$$

Lemma 5.2 (Lemma 4.2 in [2]) If the step size $\alpha$ satisfies

$$
-\psi^{\prime}\left(\lambda_{\min }(V)-2 \alpha \delta\right)+\psi^{\prime}\left(\lambda_{\min }(V)\right) \leqslant 2 \delta
$$

then $f_{1}^{\prime}(\alpha) \leqslant 0$.

Lemma 5.3 (Lemma 4.3 in [2]) Let $\rho:[0, \infty) \rightarrow(0,1]$ denote the inverse function of the restriction of $-\frac{1}{2} \psi^{\prime}(t)$ on the interval $(0,1]$, then the largest possible value of the step size of $\alpha$ satisfying (5.4) is given by

$$
\bar{\alpha}:=\frac{1}{2 \delta}(\rho(\delta)-\rho(2 \delta)) .
$$

Lemma 5.4 (Lemma 4.4 in [2]) Let $\rho$ and $\bar{\alpha}$ be the same as defined in Lemma 5.3. Then

$$
\bar{\alpha} \geqslant \frac{1}{\psi^{\prime \prime}(\rho(2 \delta))} .
$$

For the purpose of finding an upper bound for $f(\alpha)$, we need a default step size $\tilde{\alpha}$ that is the lower bound of the $\bar{\alpha}$ and consists of $\delta$.

Lemma 5.5 One has

$$
\frac{1}{\psi^{\prime \prime}(\rho(2 \delta))} \geqslant \frac{1}{1+q\left(1+\frac{4 q \delta}{q-1}\right)\left(1+q^{-1} \log \left(1+\frac{4 q \delta}{q-1}\right)\right)^{3}} .
$$


Proof To obtain the inverse function $t=\rho(s)$ of $-\frac{1}{2} \psi^{\prime}(t)$ for $t \in(0,1]$, we need to solve the equation

$$
-\psi^{\prime}(t)=-t+\left(\frac{1}{t}-\frac{1}{q}\right) e^{q\left(\frac{1}{t}-1\right)}+\frac{1}{q}=2 s .
$$

By setting $t=\rho(2 \delta)$, we have

$$
-\psi^{\prime}(t)=4 \delta
$$

Hence, we have

$$
\left(\frac{1}{t}-\frac{1}{q}\right) e^{q\left(\frac{1}{t}-1\right)}=4 \delta+t-\frac{1}{q} \leqslant 4 \delta+1-\frac{1}{q}=4 \delta+\frac{q-1}{q} .
$$

For $t \leqslant 1$ we have $1-\frac{1}{q} \leqslant \frac{1}{t}-\frac{1}{q}$. Therefore

$$
\left(1-\frac{1}{q}\right) e^{q\left(\frac{1}{t}-1\right)} \leqslant 4 \delta+\frac{q-1}{q} .
$$

This implies that

$$
e^{q\left(\frac{1}{t}-1\right)} \leqslant \frac{4 q \delta}{q-1}+1
$$

By taking the logarithm of both sides of (5.5), we obtain

$$
q\left(\frac{1}{t}-1\right) \leqslant \log \left(1+\frac{4 q \delta}{q-1}\right),
$$

and hence

$$
t=\rho(2 \delta) \geqslant \frac{1}{1+q^{-1} \log \left(1+\frac{4 q \delta}{q-1}\right)} .
$$

It follows from (4.2), (5.5) and (5.7) that

$$
\frac{1}{\psi^{\prime \prime}(\rho(2 \delta))} \geqslant \frac{1}{1+q\left(1+\frac{4 q \delta}{q-1}\right)\left(1+q^{-1} \log \left(1+\frac{4 q \delta}{q-1}\right)\right)^{3}} .
$$

This completes the proof.

In the sequel, we use the notation

$$
\tilde{\alpha}=\frac{1}{1+q\left(1+\frac{4 q \delta}{q-1}\right)\left(1+q^{-1} \log \left(1+\frac{4 q \delta}{q-1}\right)\right)^{3}},
$$

and we will use $\tilde{\alpha}$ as the default step size. By Lemma 5.4, $\bar{\alpha} \geqslant \tilde{\alpha}$.

Lemma 5.6 (Lemma 4.5 in [2]) If the step size $\alpha$ is such that $\alpha \leqslant \tilde{\alpha}$, then

$$
f(\alpha) \leqslant-\alpha \delta^{2} .
$$


The following lemma customizes the result of Lemma 5.6 for the default step size.

Lemma 5.7 If $\tilde{\alpha}$ is the default step size as given by (5.4) and $\Psi \geqslant \tau \geqslant 1$, then

$$
f(\tilde{\alpha}) \leqslant \frac{-\sqrt{\Psi}}{2+q \sqrt{2}\left(\sqrt{2}+\frac{4 q}{q-1}\right)\left(1+q^{-1} \log \left(1+\frac{4 q}{q-1} \sqrt{\frac{\Psi_{0}}{2}}\right)\right)^{3}} .
$$

Proof Using Lemma 5.6 with $\alpha=\tilde{\alpha}$ and (5.4), we have

$$
f(\tilde{\alpha}) \leqslant \frac{-\delta^{2}}{1+q\left(1+\frac{4 q \delta}{q-1}\right)\left(1+q^{-1} \log \left(1+\frac{4 q \delta}{q-1}\right)\right)^{3}} .
$$

This expresses the decrease in one inner iteration in terms of $\delta$. Since the right-hand side of the above expression is monotonically decreasing in $\delta$, we can express the decrease in terms of $\Psi:=\Psi(V)$ by Lemma 4.5 and after some elementary reductions as follows:

$$
\begin{aligned}
f(\tilde{\alpha}) & \leqslant \frac{-\delta}{\sqrt{2}+q\left(\sqrt{2}+\frac{4 q}{q-1}\right)\left(1+q^{-1} \log \left(1+\frac{4 q \delta}{q-1}\right)\right)^{3}} \\
& \leqslant \frac{-\sqrt{\Psi}}{2+q \sqrt{2}\left(\sqrt{2}+\frac{4 q}{q-1}\right)\left(1+q^{-1} \log \left(1+\frac{4 q}{q-1} \sqrt{\frac{\Psi_{0}}{2}}\right)\right)^{3}},
\end{aligned}
$$

where the last inequality follows from $\Psi_{0} \geqslant \Psi$, and therefore the proof is complete.

\subsection{Iteration Bound}

We need to count how many inner iterations are required to return to the situation where $\Psi(V) \leqslant \tau$ after a $\mu$-update. We define the value of $\Psi(V)$ after $\mu$-update as $\Psi_{0}$ and the subsequent values in the same outer iteration are denoted as $\Psi_{k}, k=$ $1,2, \cdots, K$, where $K$ denotes the total number of inner iterations in the outer iteration. Then, we have

$$
\Psi_{0} \leqslant \frac{n \theta+2 \tau+\sqrt{8 n \tau}}{2(1-\theta)}, \quad \Psi_{K-1}>\tau, \quad 0 \leqslant \Psi_{K} \leqslant \tau .
$$

Since the large-update methods use $\theta=\Theta(1)$ and $\tau=\mathcal{O}(n)$, we have $\Psi_{0}=\mathcal{O}(n)$.

According to decrease of $f(\tilde{\alpha})$, for $k=1,2, \cdots, K-1$, we obtain

$$
\begin{aligned}
\Psi_{k+1} & \leqslant \Psi_{k}-\frac{\Psi_{k}^{\frac{1}{2}}}{2+q \sqrt{2}\left(\sqrt{2}+\frac{4 q}{q-1}\right)\left(1+q^{-1} \log \left(1+\frac{4 q}{q-1} \sqrt{\frac{\Psi_{0}}{2}}\right)\right)^{3}} \\
& \leqslant \Psi_{k}-\frac{\Psi_{k}^{\frac{1}{2}}}{2+q \sqrt{2}(\sqrt{2}+8)\left(1+q^{-1} \log \left(1+8 \sqrt{\frac{\Psi_{0}}{2}}\right)\right)^{3}},
\end{aligned}
$$

where the last inequality follows by $\frac{q}{q-1} \leqslant 2$. 
The following lemma is crucial in deriving the number of inner iterations in an outer iteration.

Lemma 5.8 (Lemma 14 in [16]) Suppose $t_{0}, t_{1}, \cdots, t_{k}$ be a sequence of positive numbers such that

$$
t_{k+1} \leqslant t_{k}-\beta t_{k}^{1-\gamma}, \quad k=0,1, \cdots, K-1,
$$

where $\beta>0$ and $0<\gamma \leqslant 1$. Then $K \leqslant\left\lceil\frac{t_{0}^{\gamma}}{\beta \gamma}\right\rceil$.

Now, using Lemma 5.8, letting $t_{k}=\Psi_{k}, \gamma=\frac{1}{2}$ and

$$
\beta=\frac{1}{2+q \sqrt{2}(\sqrt{2}+8)\left(1+q^{-1} \log \left(1+8 \sqrt{\frac{\Psi_{0}}{2}}\right)\right)^{3}},
$$

we can provide the worst case upper bound for the total number of inner iterations in an outer iteration as follows:

Lemma 5.9 Let $K$ be the total number of inner iterations in the outer iteration. Then

$$
K \leqslant\left(4+2 q \sqrt{2}(\sqrt{2}+8)\left(1+q^{-1} \log \left(1+8 \sqrt{\frac{\Psi_{0}}{2}}\right)\right)^{3}\right) \Psi_{0}^{\frac{1}{2}} .
$$

Now, we can derive the iteration complexity of our algorithm.

Theorem 5.1 Given that $\tau=\mathcal{O}(n)$ and $\theta=\Theta(1)$, which are characteristics of the large-update methods, Algorithm 3.1 will obtain an $\epsilon$-approximate solution of problems (P) and (D) in at most $\mathcal{O}\left(\sqrt{n} \log n \log \frac{n}{\epsilon}\right)$ iterations.

Proof It is well known that the number of outer iterations is bounded above by $\frac{1}{\theta} \log \left(\frac{n}{\epsilon}\right)$ [Lemma II.17 in [18]]. By multiplying the number of outer iterations and the number of inner iterations we get an upper bound for the total number of iterations, namely,

$$
\begin{aligned}
& \frac{4+}{q(16 \sqrt{2}+4)\left(1+q^{-1} \log \left(1+8 \sqrt{\frac{n \theta+2 \tau+\sqrt{8 n \tau}}{4(1-\theta)}}\right)\right)^{3}} \theta \\
& \times\left(\frac{n \theta+2 \tau+\sqrt{8 n \tau}}{2(1-\theta)}\right)^{\frac{1}{2}} \log \left(\frac{n}{\epsilon}\right) .
\end{aligned}
$$

Choosing $q=\log \left(1+8 \sqrt{\frac{n \theta+2 \tau+\sqrt{8 n \tau}}{4(1-\theta)}}\right)=\mathcal{O}(\log n)$, the iteration bound becomes

$$
\mathcal{O}\left(\sqrt{n} \log n \log \frac{n}{\epsilon}\right) .
$$




\section{Numerical Example}

In this section, we illustrate that the proposed algorithm is promising by a numerical experiment. The numerical experiment is implemented on MATLAB 7.8 under Windows XP. We consider the CQSDO problems whose primal-dual pair of (P) and (D) have the following date:

$$
\begin{array}{rlrl}
A_{1} & =\left[\begin{array}{cccc}
0 & 1 & 0 & 0 \\
1 & 2 & 0 & -1 \\
0 & 0 & -2 & -1 \\
0 & -1 & -1 & -2
\end{array}\right], & A_{2}=\left[\begin{array}{cccc}
0 & 0 & -2 & 0 \\
0 & 2 & 1 & 2 \\
-2 & 1 & -2 & 1 \\
0 & 2 & 1 & 2
\end{array}\right], \\
A_{3} & =\left[\begin{array}{cccc}
2 & 2 & -1 & -1 \\
2 & 0 & 2 & 1 \\
-1 & 2 & 0 & 1 \\
-1 & 1 & 1 & -2
\end{array}\right], \\
C & =\left[\begin{array}{cccc}
2 & 3 & -3 & -1 \\
3 & 4 & 3 & 2 \\
-3 & 3 & -4 & 1 \\
-1 & 2 & 1 & -2
\end{array}\right], & Q=\left[\begin{array}{llll}
1 & 0 & 0 & 0 \\
0 & 1 & 0 & 0 \\
0 & 0 & 1 & 0 \\
0 & 0 & 0 & 1
\end{array}\right], \quad b=\left[\begin{array}{c}
-2 \\
2 \\
0
\end{array}\right] .
\end{array}
$$

One may easily verify that $X^{0}=I$ is feasible for (P) and $y=(1,1,1)^{T}$ and $S^{0}=I$ is feasible for (D). We obtain a primal-dual optimal solution

$$
\begin{aligned}
X^{*} & =\left[\begin{array}{cccc}
0.0574 & -0.0368 & -0.0554 & -0.0304 \\
-0.0368 & 0.0648 & 0.0536 & 0.1540 \\
-0.0554 & 0.0536 & 0.2056 & 0.1688 \\
-0.0304 & 0.1540 & 0.1688 & 0.4996
\end{array}\right], \quad y^{*}=\left[\begin{array}{l}
0.8458 \\
1.0559 \\
0.9747
\end{array}\right], \\
S^{*} & =\left[\begin{array}{cccc}
0.1081 & 0.1681 & 0.0311 & -0.0557 \\
0.1681 & 0.2615 & 0.0483 & -0.0867 \\
0.0311 & 0.0483 & 0.0089 & -0.0160 \\
-0.0557 & -0.0867 & -0.0160 & 0.0287
\end{array}\right] .
\end{aligned}
$$

We have a tolerance $\epsilon=10^{-5}$ and $\theta=\frac{1}{\sqrt{4}}$ that the algorithm reaches this solution in 23 iterations.

\section{Conclusion}

We have analyzed a primal-dual path-following interior-point algorithm for CQSDO based on a new kernel function and derived the currently best known iteration bound for the algorithm with large-update method, $\mathcal{O}\left(\sqrt{n} \log n \log \frac{n}{\epsilon}\right)$. The numerical test is an interesting topic for investigating the behavior of the algorithm.

Acknowledgements The authors gratefully acknowledge the help of the editor and anonymous referees in improving the readability of the paper. 


\section{References}

[1] Amini, K., Haseli, A.: A new proximity function generating the best known iteration bounds for both large-update and small-update interior-point methods. ANZIAM J. 49(2), 259-270 (2007)

[2] Bai, Y.Q., El Ghami, M., Roos, C.: A comparative study of kernel functions for primal-dual interiorpoint algorithms in linear optimization. SIAM J. Optim. 15(1), 101-128 (2004)

[3] Bai, Y.Q., Guo, J., Roos, C.: A new kernel function yielding the best known iteration bounds for primal-dual interior-point algorithms. Acta Math. Sin. Engl. Ser. 25(12), 2169-2178 (2009)

[4] Bai, Y.Q., Roos, C.: A polynomial-time algorithm for linear optimization based a new simple kernel function. Optim. Methods Softw. 18(6), 631-646 (2003)

[5] Bai, Y.Q., Lesaja, G., Roos, C., Wang, G.Q., El Ghami, M.: A class of large-update and small-update primal-dual interior-point algorithms for linear optimization. J. Optim. Theory Appl. 138(3), 341359 (2008)

[6] Bai, Y.Q., El Ghami, M., Roos, C.: A new efficient large-update primal-dual interior-point method based on a finite barrier. SIAM J. Optim. 13(3), 766-782 (2003)

[7] Bai, Y.Q., El Ghami, M., Roos, C.: Kernel function based algorithms for semidefinite optimization. Int. J. RAIRO, Oper. Res. 43(2), 189-199 (2009)

[8] Bai, Y.Q., Wang, F.Y., Luo, X.W.: A polynomial-time interior-point algorithm for convex quadratic semidefinite optimization. RAIRO Oper. Res. 44, 251-265 (2010)

[9] Horn, R.A., Johnson, C.R.: Topics in Matrix Analysis. Cambridge University Press, Cambridge (1991)

[10] Kheirfam, B.: Primal-dual interior-point algorithm for semidefinite optimization based on a new kernel function with trigonometric barrier term. Numer. Algorithms 61(4), 659-680 (2012)

[11] Kojima, M., Shida, M., Hara, S.: Interior point methods for the monotone semidefinite linear complementarity problem in symmetric matrices. SIAM J. Optim. 7, 86-125 (1997)

[12] Nesterov, Y.E., Todd, M.J.: Self-scaled barriers and interior-point methods for convex programming. Math. Oper. Res. 22(1), 1-42 (1997)

[13] Nesterov, Y.E., Todd, M.J.: Primal-dual interior-point methods for self-scaled cones. SIAM J. Optim. 8(2), 324-364 (1998)

[14] Nie, J.W., Yuan, Y.X.: Apotential reduction algorithm for an extended SDP problem. Sci. China Ser. A 43(1), 35-46 (2000)

[15] Nie, J.W., Yuan, Y.X.: A predictor-corrector algorithm for QSDP combining Dikin-type and Newton centering steps. Ann. Oper. Res. 103, 115-133 (2001)

[16] Peng, J., Roos, C., Terlaky, T.: Self-regular functions and new search directions for linear and semidefinite optimization. Math. Program. 93(1), 129-171 (2002)

[17] Peng, J., Roos, C., Terlaky, T.: Self-regularity: A New Paradigm for Primal-Dual Interior-Point Algorithms. Princeton University Press, Princeton (2002)

[18] Roos, C., Terlaky, T., Vial, J.-P.: Theory and Algorithms for Linear Optimization. An Interior-Point Approach. Wiley, Chichester (1997)

[19] Toh, K.C.: Inexact primal-dual path-following algorithms for a convex quadratic SDP. Math. Program. 112(1), 221-254 (2008)

[20] Wang, G.Q., Bai, Y.Q.: Primal-dual interior-point algorithm for convex quadratic semi-definite optimization. Nonlinear Anal. 71(7-8), 3389-3402 (2009)

[21] Wang, G.Q., Zhu, D.T.: A unified kernel function approach to primal-dual interior-point algorithms for convex quadratic SDO. Numer. Algorithms 57(4), 537-558 (2011)

[22] Wolkowicz, H., Saigal, R., Vandenberghe, L.: Handbook of Semidefinite Programming, Theory, Algorithm, and Applications. Kluwer Academic, Dordrecht (2000)

[23] Zhang, M.W.: A large-update interior-point algorithm for convex quadratic semidefinite optimization based on a new kernel function. Acta Math. Sin. Engl. Ser. 28(11), 2313-2328 (2012) 